\title{
Off-axis spin orientation in goethite nanoparticles
}

\author{
Brok, Erik; Lefmann, Kim; Nilsen, Gøran Jan; Kure, Mathias; Frandsen, Cathrine
}

Published in:

Physical Review B

Link to article, DOI:

10.1103/PhysRevB.96.104426

Publication date:

2017

Document Version

Publisher's PDF, also known as Version of record

Link back to DTU Orbit

Citation (APA):

Brok, E., Lefmann, K., Nilsen, G. J., Kure, M., \& Frandsen, C. (2017). Off-axis spin orientation in goethite nanoparticles. Physical Review B, 96(10), [104426 ]. https://doi.org/10.1103/PhysRevB.96.104426

\section{General rights}

Copyright and moral rights for the publications made accessible in the public portal are retained by the authors and/or other copyright owners and it is a condition of accessing publications that users recognise and abide by the legal requirements associated with these rights.

- Users may download and print one copy of any publication from the public portal for the purpose of private study or research.

- You may not further distribute the material or use it for any profit-making activity or commercial gain

- You may freely distribute the URL identifying the publication in the public portal

If you believe that this document breaches copyright please contact us providing details, and we will remove access to the work immediately and investigate your claim. 
PHYSICAL REVIEW B 96, 104426 (2017)

\title{
Off-axis spin orientation in goethite nanoparticles
}

\author{
Erik Brok ${ }^{*}$ \\ National Institute of Standards and Technology, Gaithersburg, Maryland 20899, USA \\ and Department of Materials Science and Engineering, University of Maryland, College Park, Maryland 20740, USA
}

Kim Lefmann

Nano-Science Center, Niels Bohr Institute, University of Copenhagen, DK-2100 Copenhagen Ø, Denmark

Gøran Jan Nilsen ${ }^{\dagger}$

Institut Max von Laue Paul Langevin, F-38042 Grenoble, France

Mathias Kure and Cathrine Frandsen

Department of Physics, Technical University of Denmark, DK-2800 Kgs Lyngby, Denmark

(Received 5 July 2017; revised manuscript received 7 September 2017; published 19 September 2017)

\begin{abstract}
Neutron diffraction is a powerful technique for determining the magnetic structure of antiferromagnetic materials. However, for some of these, determining the detailed magnetic structure remains a challenge. In goethite $(\alpha-\mathrm{FeOOH})$ the antiferromagnetic unit cell coincides with the chemical unit cell and, consequently, nuclear and magnetic diffraction peaks occur at the same positions. Analysis of diffraction data from goethite is further complicated by finite-size peak broadening, resulting from goethite commonly occurring in nanocrystalline form. For these reasons, determining the magnetic structure of goethite has been challenging, and few detailed studies have been published. Even today, not all aspects of the magnetic structure are well established. Here, we investigate the magnetic structure of three samples of goethite nanoparticles with polarized neutron powder diffraction (xyz-polarization analysis). Two samples consist of acicular goethite particles that are approximately $40 \mathrm{~nm}$ long and with different thicknesses, and one sample consists of pseudo-spherical particles with a diameter of approximately $5 \mathrm{~nm}$. The larger particles consist of several crystallites whereas the 5-nm particles are mostly single crystalline. The polarization analysis enables us to separate magnetic scattering from nuclear and spin-incoherent scattering, resulting in data that can readily be analyzed. For the two samples with the larger particle size, we find nuclear correlation lengths in the [100] direction that are approximately $3 \mathrm{~nm}$ longer than magnetic correlation lengths, indicating a magnetically disordered layer perpendicular to the antiferromagnetic modulation direction. We find no evidence of a magnetically disordered surface layer in the 5-nm particles. We find the magnetic structure to be antiferromagnetic but, in contrast to most previous studies, we find the spin orientation in all three samples to make an angle of $28-30^{\circ}$ with respect to the crystallographic $b$ axis.
\end{abstract}

DOI: 10.1103/PhysRevB.96.104426

\section{INTRODUCTION}

Despite the fact that antiferromagnetic materials do not possess a net magnetization, they are of considerable importance in applications, usually in combination with ferro- or ferrimagnetic materials exchange coupled to the antiferromagnet. Of particular interest are nanocomposites, where different types of magnetic materials are combined on the nanoscale, e.g., in core-shell structures or by embedding one material in a matrix of another. This is a promising and active field of research [1-10].

The technique of choice for determining the magnetic structure of antiferromagnetic materials is neutron diffraction. The antiferromagnetic unit cell is often larger than the chemical unit cell and, therefore, the antiferromagnetic diffraction peaks commonly appear at different diffraction angles than the peaks

\footnotetext{
*Present address: Nano-Science Center, Niels Bohr Institute, University of Copenhagen, DK-2100 Copenhagen Ø, Denmark; e.brok@nbi.ku.dk

${ }^{\dagger}$ Present address: ISIS Facility, Rutherford Appleton Laboratory, Chilton, Didcot, Oxon OX11 0QX, United Kingdom.
}

from the chemical structure. This facilitates magnetic structure determination.

When the particle size is decreased to the nanoscale, the magnetic structure can differ from that of the corresponding bulk material because of broken exchange-bonds at the particle surface and because of surface contributions to the magnetic anisotropy [11-14]. The change in magnetic structure as well as dynamics in magnetic nanoparticles can be of crucial importance for their applications and, hence, determining the magnetic structure of nanoparticles is important. However, doing this by neutron diffraction can be complicated due to overlapping diffraction peaks from finite-size peak broadening [14]. Furthermore, neutron scattering studies of nanoparticles are often hampered by a large background from spin-incoherent scattering by hydrogen in water adsorbed at the particle surfaces. This problem is further exacerbated if the material under study has hydrogen in the structure.

Goethite $(\alpha-\mathrm{FeOOH})$ is a commonly occurring mineral that crystallizes in the orthorhombic Pnma space group. Below a Néel temperature of about $400 \mathrm{~K}$, the $\mathrm{Fe}^{3+}$ spins order antiferromagnetically. In nonstoichiometric goethite and in goethite samples with small crystallite sizes, the 
Néel temperature is found to be reduced to somewhere in the range $300 \mathrm{~K}-400 \mathrm{~K}$ [15-17] or even lower [18]. The antiferromagnetic unit cell is congruent with the chemical unit cell $[15,19,20]$, and this has the consequence that the antiferromagnetic diffraction peaks occur at the same positions as peaks from the chemical structure. This makes investigations of the magnetic structure of goethite with neutron diffraction particularly challenging, although a reasonable determination can be achieved by comparing the intensities of diffraction peaks $[15,18-20]$ to models for the chemical and magnetic structure.

Goethite commonly occurs in nanocrystalline form, with the magnetic properties of even relatively large goethite particles being dominated by fluctuations in weakly interacting grains which are only a few nanometers in size [21,22]. Natural as well as synthetic goethite samples exhibit varying degrees of nonstoichiometry with resulting vacancies on the $\mathrm{Fe}$ sites of potential importance for the magnetic structure [17,23]. Besides crystallinity, stoichiometry, and defects or impurities, other sample-dependent factors such as particle size and morphology could affect the magnetic structure differently for different samples.

The difficulties associated with the magnetic structure of goethite and, in particular, with sample-dependent contributions, means that there is some discrepancy between the findings in different studies of the magnetic structure and dynamics of goethite. Zepeda-Alarcon et al. [18] used Rietveld refinement of neutron powder diffraction data to determine the magnetic structure of two natural samples of goethite. They obtained good diffraction patterns of their sample A, which consisted of well-crystallized particles with a diameter of about $96 \mathrm{~nm}$ (assuming spherical particles), and investigated the magnetic structure using Rietveld refinement of the diffraction data. Their diffraction patterns from a sample with a smaller particle size $(\approx 10 \mathrm{~nm})$ has a much lower signal-to-noise ratio and substantial peak broadening, and refining the details of the magnetic structure is probably less reliable than for the sample with larger crystallites. This shows that determining the magnetic structure of goethite with a small particle size can be challenging using conventional methods.

Polarized neutron diffraction can be used to study the magnetic structure of antiferromagnetic nanoparticles by separating magnetic and nuclear scattering and by further removing spin-incoherent scattering, as has been previously demonstrated [14]. The ability to separate the different scattering contributions is even more useful in the case of goethite because of the perfect overlap of the chemical and magnetic structures and because of the hydrogen in the structure. Here we use polarized neutron powder diffraction to study the magnetic structure of three samples of goethite in nanocrystalline form with different particle sizes. In the sample with the smallest particle size, the particle diameter $(\approx 5 \mathrm{~nm})$ is on the order of the crystallite size. In the case of the two samples with larger particle size (length of $\approx 40 \mathrm{~nm}$ thicknesses from $\approx 3 \mathrm{~nm}$ to $\approx 12 \mathrm{~nm}$ ), the crystalline size is smaller than the particle size, and the particles consist of several interacting grains. For these samples, we thus study the magnetic structure in interacting nanoscale grains of goethite.
TABLE I. Approximate positions of $\mathrm{Fe}$ atoms in the goethite structure.

\begin{tabular}{lcccc}
\hline \hline Atom & $x$ & $y$ & $z$ & Spin \\
\hline $\mathrm{Fe}^{(1)}$ & 0.146 & $\frac{3}{4}$ & 0.952 & + \\
$\mathrm{Fe}^{(2)}$ & 0.854 & $\frac{1}{4}$ & 0.048 & - \\
$\mathrm{Fe}^{(3)}$ & 0.646 & $\frac{3}{4}$ & 0.548 & + \\
$\mathrm{Fe}^{(4)}$ & 0.354 & $\frac{1}{4}$ & 0.452 & - \\
\hline \hline
\end{tabular}

\section{CHEMICAL AND MAGNETIC STRUCTURE OF GOETHITE}

The goethite structure belongs to the Pnma space group ${ }^{1}$ with unit cell parameters $a \approx 9.95 \AA, b \approx 3.02 \AA$, and $c \approx$ 4.60 $\AA$. There are four formula units in the unit cell, with atoms occupying positions $\pm\left(\tilde{x}, \frac{1}{4}, \tilde{z}\right)$ and $\pm\left(\frac{1}{2}-\tilde{x}, \frac{3}{4}, \frac{1}{2}+\tilde{z}\right)$, with $\tilde{x}$ and $\tilde{z}$ taking different values for $\mathrm{Fe}, \mathrm{H}, \mathrm{O}_{I}$, and $\mathrm{O}_{I I}$ atoms. For the Fe atoms, $\tilde{x} \approx 0.85$ and $\tilde{z} \approx 0.048[15,18,20]$ lead to the approximate positions given in Table I. The direction of antiferromagnetic modulation is consistently found to be along the crystallographic $a$ axis with a $(+-+-)$ alignment of $\mathrm{Fe}$ spins 1-4 as depicted in Fig. 1 [15,18,20]. In most studies, the spins are found to be oriented along the $b$ axis and the magnetic space group is then $P_{n m a}$ [15,18,20]. However, Coey et al. [19] found the antiferromagnetically ordered spins to be oriented away from the $b$ axis by an angle of $13^{\circ}$, resulting in the magnetic space group $P 2_{1}^{\prime} / c$.

\footnotetext{
${ }^{1}$ In some literature $[16,18,20]$ the goethite structure is described in terms of the equivalent Pbnm space group.
}

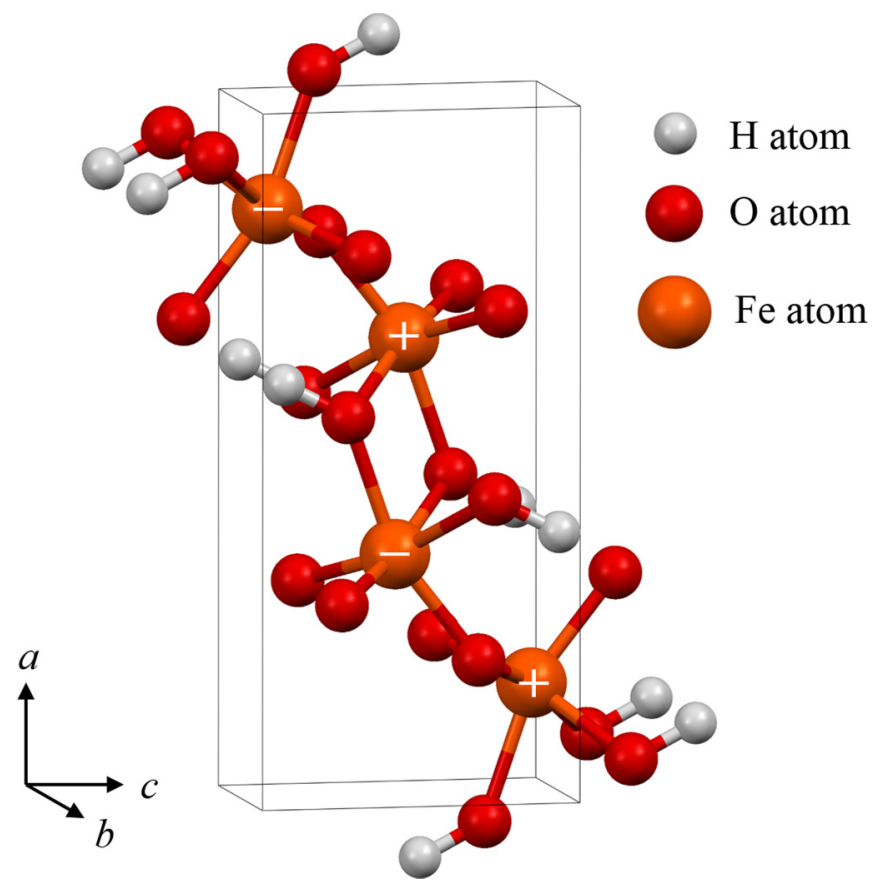

FIG. 1. Magnetic structure of goethite ( $\alpha$-FeOOH). Only the four $\mathrm{Fe}$ atoms in the unit cell (large orange balls) and their associated $\mathrm{O}$ atoms (smaller red balls) and hydrogens (grey balls) are shown. 
TABLE II. The first six nonzero nuclear and magnetic structure factors. The $\{h k l\}$ indices refer to the orthorhombic Pnma structure. The structure factors were calculated using Eqs. (1) and (2) with $b_{\mathrm{O}}=$ $5.805 \mathrm{fm}, b_{\mathrm{H}}=-3.7409 \mathrm{fm}, b_{\mathrm{Fe}}=9.45 \mathrm{fm}$, and the atomic positions determined from the $15 \mathrm{~K}$ data for sample A in Zepeda-Alarcon et al. [18]. The uncertainty is on the last digit. Also given is the multiplicity $j$ of each reflection.

\begin{tabular}{lcccc}
\hline \hline$\{h k l\}$ & $q\left(\AA^{-1}\right)$ & $F_{\mathrm{N}}$ & $F_{\mathrm{M}}$ & $j$ \\
\hline$\{200\}$ & 1.264 & 1.817 & 3.844 & 2 \\
$\{001\}$ & 1.367 & 0 & 1.191 & 2 \\
$\{101\}$ & 1.506 & 3.691 & 3.020 & 4 \\
$\{201\}$ & 1.862 & 1.744 & 0.313 & 4 \\
$\{010\}$ & 2.081 & 0 & 3.975 & 2 \\
$\{301\}$ & 2.337 & 2.410 & 1.426 & 4 \\
\hline \hline
\end{tabular}

\section{A. Structure factors}

For a reflection with Miller indices $h k l$, the structure factor for nuclear scattering is defined as

$$
F_{\mathrm{N}}(h k l)=\sum_{j} b_{j} \exp \left[2 \pi i\left(h x_{j}+k y_{j}+l z_{j}\right)\right],
$$

where the sum is over all atoms in the unit cell and $b_{j}$ is the scattering length of atom $j$ with coordinates $\left(x_{j}, y_{j}, z_{j}\right)$. Similarly, the magnetic structure factor for a two-sublattice antiferromagnet is

$$
F_{\mathrm{M}}(h k l)=\sum_{j} p_{j} \exp \left[2 \pi i\left(h x_{j}+k y_{j}+l z_{j}\right)\right],
$$

where the sum is over the magnetic atoms and $p$ describes the sign of the spin. For goethite, the sum is over the four $\mathrm{Fe}$ atoms in the unit cell and $p$ takes the values $+1,-$ $1,+1,-1$ for atoms 1-4, respectively. Nuclear and magnetic structure factors for goethite were calculated using the atomic positions determined in Ref. [18] by Rietveld refinement of neutron powder diffraction data from a goethite sample with a 97-nm crystallite size. Separate structure factors were calculated using the atomic positions determined at $15 \mathrm{~K}$ and $300 \mathrm{~K}$, and these were in turn used to analyze our data at low temperature $(1.5 \mathrm{~K})$ and high temperature $(150 \mathrm{~K}$ and $300 \mathrm{~K})$, respectively. The first six nonzero structure factors determined from the $15 \mathrm{~K}$ data are given in Table II. Note that the $\{001\}$ and $\{010\}$ reflections are purely magnetic, while the rest will have both magnetic and nuclear intensities.

\section{B. Scattering cross sections}

For a nuclear $h k l$ reflection, the cross section for scattering of neutrons with wavelength $\lambda$ will be

$$
\sigma_{\mathrm{N}, \mathrm{hkl}}=\frac{N}{V_{0}} \frac{\lambda^{3}}{4 L_{\theta_{h k l}}} j_{h k l}\left|F_{\mathrm{N}, h k l}\right|^{2},
$$

where $N$ is the number of unit cells, $V_{0}$ is the unit cell volume, $j_{h k l}$ is the multiplicity of the reflection, and $L_{\theta_{h k l}}=$ $\sin \left(\theta_{h k l}\right) \sin \left(2 \theta_{h k l}\right)$ is the Lorentz factor which depends on the scattering angle $2 \theta_{h k l}$. Similarly, the cross section for a magnetic $h k l$ reflection will be [24]

$$
\sigma_{M, h k l}=\frac{N}{V_{0}} \frac{\lambda^{3}}{4 L_{\theta_{h k l}}} j_{h k l}\left(\frac{\gamma r_{0}}{2}\right)^{2} g^{2} f^{2}(q)\left|F_{\mathrm{M}, h k l}\right|^{2}\left|\mathbf{M}_{\perp, h k l}\right|^{2},
$$

where $\gamma=1.913$ is the gyromagnetic ratio, $r_{0}=2.8179 \mathrm{fm}$ is the classical electron radius, $g$ is the Landé factor, $f(q)$ is the magnetic form factor, and $\mathbf{M}_{\perp, h k l}$ is the (unit less) microscopic magnetization perpendicular to q. $M_{\perp, h k l}=\left|\mathbf{M}_{\perp, h k l}\right|$ can be found from the ratio of the measured magnetic and nuclear cross sections at the same $h k l$ :

$$
M_{\perp, h k l}^{2}=\frac{\sigma_{M, h k l}}{\sigma_{N, h k l}} \frac{\left|F_{\mathrm{N}, h k l}\right|^{2}}{\left|F_{\mathrm{M}, h k l}\right|^{2}\left(\frac{\gamma r_{0}}{2}\right)^{2} g^{2} f^{2}\left(q_{h k l}\right)},
$$

i.e., the size of the magnetic moment in the $b c$ plane can be determined from the $\{200\}$ cross sections and the $\{101\}$ cross sections provide the size of the magnetic moment perpendicular to [101]. If the spins are oriented along the $b$ axis $[15,18,20]$, both the $\{200\}$ and $\{101\}$ cross sections give the full size of the magnetic moment and we should expect to find $M_{\perp, 200}=M_{\perp, 101}$. In this case, we should find zero intensity at the $\{010\}$ position. The ratio between $M_{\perp, 200}$ and $M_{\perp, 101}$ can be found directly from the ratio of the magnetic $\{200\}$ and $\{101\}$ cross sections:

$$
M_{\perp, \text { rel }}^{2}=\frac{M_{\perp, 200}^{2}}{M_{\perp, 101}^{2}}=\frac{\sigma_{M, 200}}{\sigma_{M, 101}} \frac{j_{101} f^{2}\left(q_{101}\right)\left|F_{\mathrm{M}, 101}\right|^{2} L_{\theta_{200}}}{j_{200} f^{2}\left(q_{200}\right)\left|F_{\mathrm{M}, 200}\right|^{2} L_{\theta_{101}}} .
$$

If the measured $M_{\perp, \text { rel }}$ is larger than unity, the spins must be rotated away from the $b$ axis in the $b c$ plane (making $M_{\perp, 101}$ smaller while not changing $\left.M_{\perp, 200}\right)$. This is the structure observed by Coey et al. [19]. If, on the other hand, $M_{\perp, \text { rel }}$ was smaller than unity, the spins would have to be rotated towards the $a$ axis. Assuming that the spins are in the $b c$ plane, $M_{\perp, 200}=M$ ( $M$ being the size of the full moment $)$ and

$$
M_{\perp, 101}=M\left(1-\sin ^{2}(\alpha) \cos ^{2}(\gamma)\right)^{\frac{1}{2}},
$$

where $\alpha$ is the spin rotation angle from the $b$ axis and $\gamma$ is the angle between [101] and [001], i.e., $\tan (\gamma)=c / a$. Now $\alpha$ can be found from

$$
\sin ^{2}(\alpha)=\frac{1-\left(\frac{M_{\perp, 101}}{M_{\perp, 200}}\right)^{2}}{\cos ^{2}(\gamma)},
$$

where the fraction in the numerator is determined from the measured cross sections through Eq. (6).

\section{EXPERIMENTAL}

\section{A. Samples}

Three powder samples of goethite with different particle sizes were obtained from NanoChemonics Inc. ${ }^{2}$

\footnotetext{
${ }^{2}$ The identification of any commercial product or trade name does not imply endorsement or recommendation by the National Institute of Standards and Technology.
} 
The samples, named G1, G2, and G3 have previously been characterized by $\mathrm{x}$-ray diffraction, transmission electron microscopy and Mössbauer spectroscopy $[22,25]$.

The G1 sample consists of pseudospherical particles with a diameter of about $5 \mathrm{~nm}$. The crystallite size, determined by broadening of $\mathrm{x}$-ray peaks $(3.9 \mathrm{~nm}, 8.0 \mathrm{~nm}$, and $4.0 \mathrm{~nm}$ along [100], [010], and [001], respectively [25]), is similar to the particle diameter, confirming that the particles typically consist of only one or very few grains, making the particles nearly monocrystalline. This is also reflected in the magnetic properties of G1, which were found to resemble single particle behavior [22].

Samples G2 and G3 consist of significantly larger particles with an acicular shape. The G2 and G3 particles are of similar length $(\approx 40 \mathrm{~nm})$ while the thickness varies between 3-10 nm for G2 and 5-12 nm for G3. X-ray diffraction shows that the crystallite size of G2 and G3 is significantly smaller than the particle dimensions $(7.6 \mathrm{~nm} \times 23.9 \mathrm{~nm} \times 6.7 \mathrm{~nm}$ and $7.8 \mathrm{~nm} \times 24.9 \mathrm{~nm} \times 9.5 \mathrm{~nm}$ along the crystallographic axes for G2 and G3, respectively [25]) and the particles thus consist of several crystallites and the magnetic properties can be expected to be characteristic of interacting goethite grains.

\section{B. Polarized neutron diffraction experiment}

Polarized neutron powder diffraction experiments were performed at the D7 instrument at Institut Laue-Langevin, Grenoble, France. The experiments were performed using $3.1 \AA$ neutrons and an instrument configuration identical to that used in a recent experiment on $\mathrm{NiO}$ nanoparticles [14] resulting in a $q$ range of approximately $0.26-3.82 \AA^{-1}$. For more details about the D7 instrument see Refs. [26,27]. The three samples were loaded in hollow-cylinder aluminum sample holders with $20 \mathrm{~mm}$ outer diameter, and $18 \mathrm{~mm}$ (G1 and G2) or $19 \mathrm{~mm}(\mathrm{G} 3)$ inner diameter. The sample masses loaded in the holders were $1.881 \mathrm{~g}, 1.705 \mathrm{~g}$, and $0.833 \mathrm{~g}$ for samples $\mathrm{G} 1, \mathrm{G} 2$, and G3, respectively. The samples were placed in a standard orange cryostat and measured at $1.5 \mathrm{~K}$ and $300 \mathrm{~K} . \mathrm{G} 2$ and G3 were measured for approximately $9 \mathrm{~h}$ at each temperature. The corresponding measurement times were approximately $13 \mathrm{~h}$ for $\mathrm{G} 1$, which was also measured at $150 \mathrm{~K}$ for approximately $9 \mathrm{~h}$. Cadmium, empty can, vanadium, and quartz were measured for background subtraction (cadmium, empty), detector efficiency calibration and absolute scaling (vanadium), and the imperfect polarization of the instrument (quartz). The data was reduced and calibrated, and nuclear, magnetic, and spin-incoherent scattering were separated.
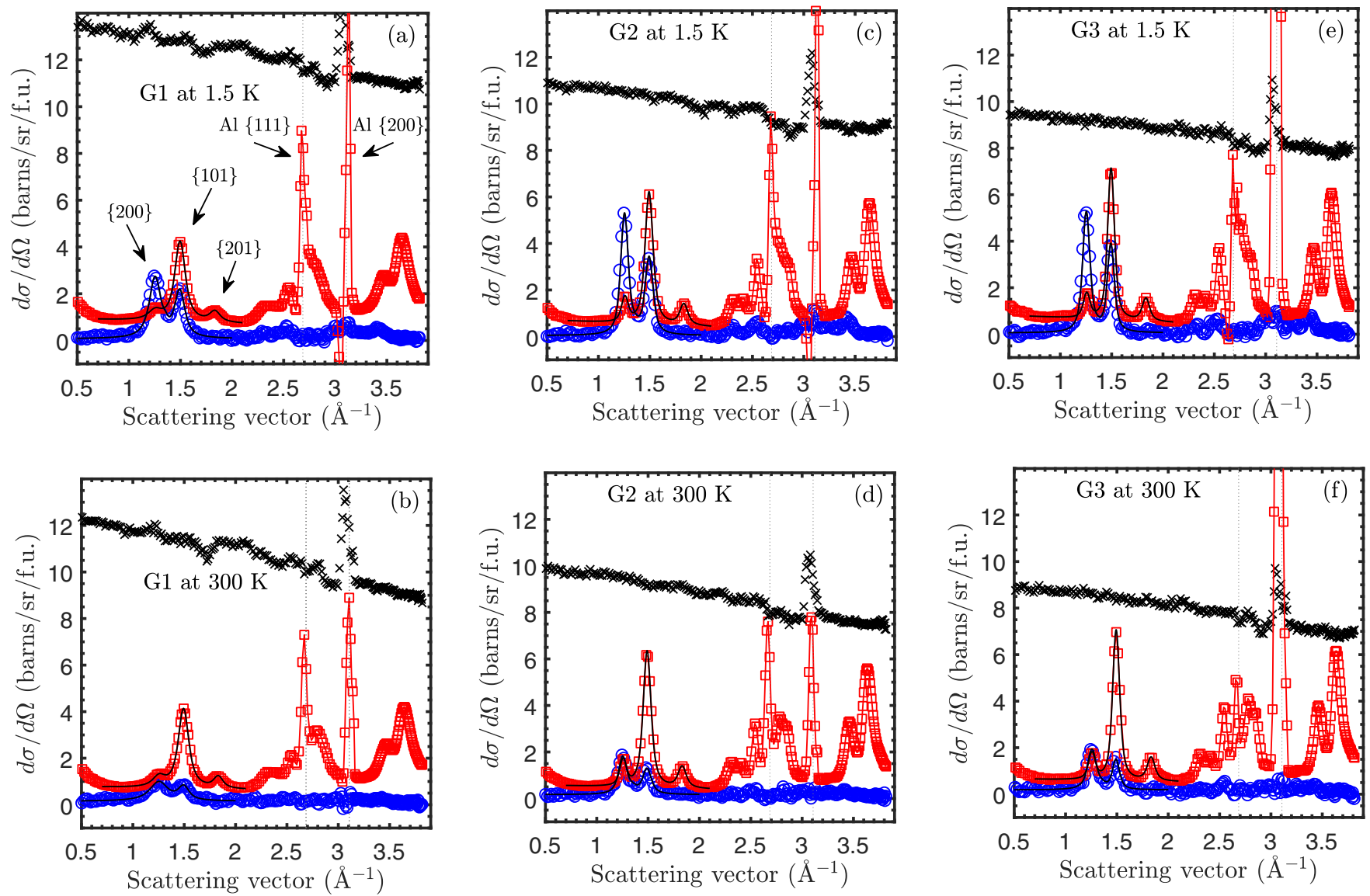

FIG. 2. Separated magnetic (o), nuclear $(\square)$, and spin-incoherent (x) cross sections for the three goethite samples measured at $1.5 \mathrm{~K}$ and $300 \mathrm{~K}$. The dashed vertical lines show the position of the $\mathrm{Al}\{111\}$ and $\{200\}$ reflections. The full black lines show the fits described later in the text. The error bars (standard deviations) are smaller than the size of the points with the exception of a few points in the spin-incoherent signal at the $\mathrm{Al}\{111\}$ position. 


\section{RESULTS}

The separated nuclear-, magnetic-, and spin-incoherent scattering cross sections measured at $1.5 \mathrm{~K}$ and $300 \mathrm{~K}$ are shown in Fig. 2 for all three samples. Overall, the separation of the three scattering contributions works well despite the very high level of spin-incoherent scattering. The spin-incoherent scattering is mostly smooth except for some structure at the position of strong nuclear reflections, most notably at the positions of the $\mathrm{Al}\{111\}$ and $\{200\}$ reflections from the sample holder. This is an artifact of the correction for imperfect neutron polarization. The spin incoherent scattering of goethite comes almost exclusively from hydrogen, which has a spin incoherent cross section of 80 barns, which translates to 6.4 barns/sr/f.u. (f.u. = formula unit). The measured levels of spin-incoherent scattering is significantly larger, between
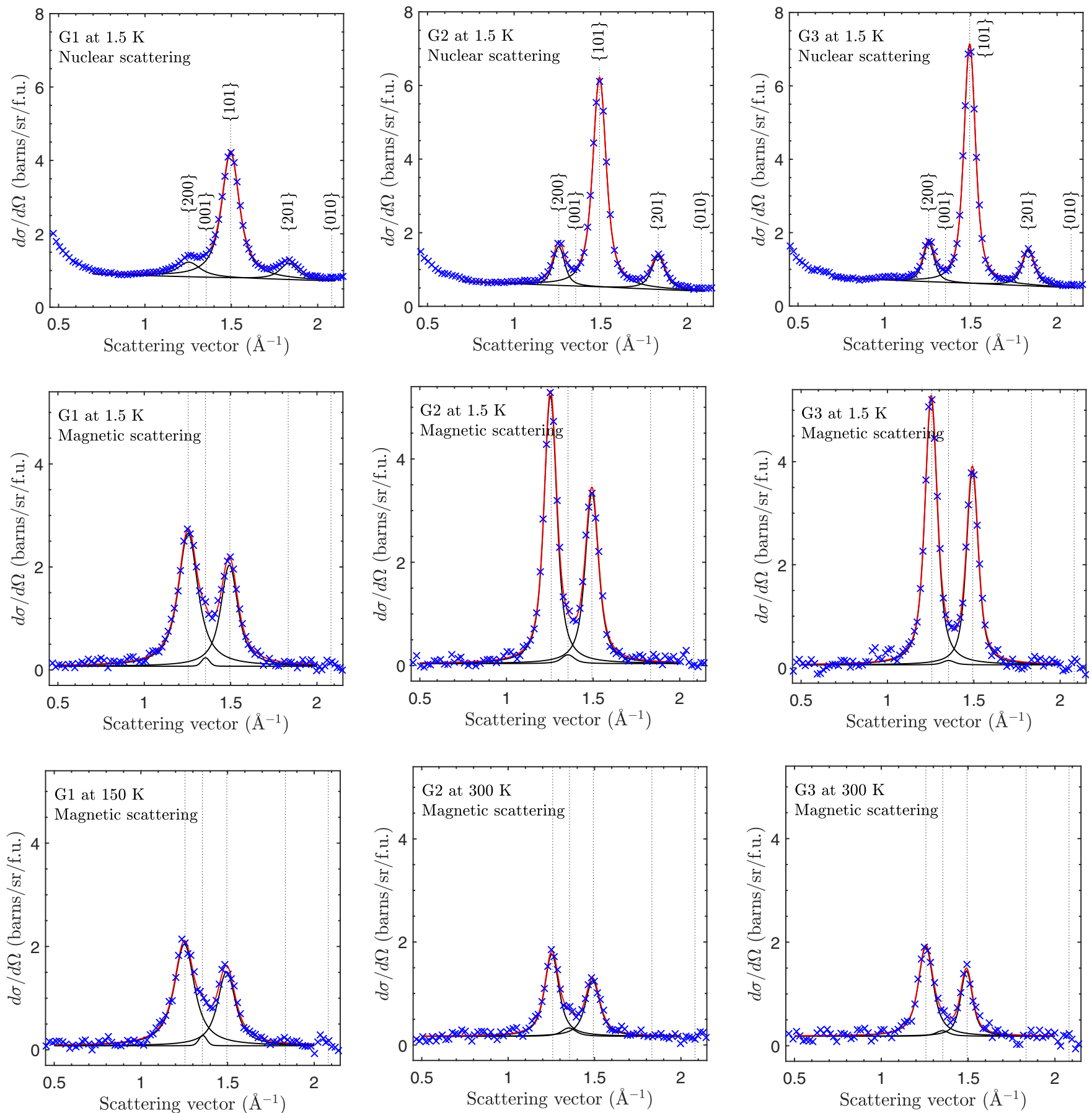

FIG. 3. Voigtian fits to the polarized powder diffraction data. Fits to the nuclear cross sections obtained at $1.5 \mathrm{~K}$ are shown in the top row. Fits to the magnetic cross sections are shown for the data obtained at $1.5 \mathrm{~K}$ in the middle row and at $150 \mathrm{~K}(\mathrm{G} 1)$ or $300 \mathrm{~K}(\mathrm{G} 2$ and G3) in the bottom row. The full black lines show the individual fitted peaks (plus background) and the red lines show the total fit. The Miller indices of the peaks are indicated in the plots of the nuclear fits (note that $\{001\}$ and $\{010\}$ are purely magnetic). The error bars (standard deviations) are smaller than the size of the points. 
7 and 14 barns/sr/f.u. and can be associated with excess water in the structure or adsorbed on the surface of the particles, leading to a larger spin-incoherent scattering for particles with larger relative surface area, i.e., smaller particles.

The nuclear scattering has diffraction peaks at the expected positions for the goethite structure as well as the two strong reflections from the Al sample holder. The diffraction peaks from the sample are significantly broadened due to the finite crystallite size. The background in the nuclear signal comes from nuclear-incoherent scattering due to variations in isotopes and structural imperfections. Fe has an isotopeincoherent cross section of 0.40 barns corresponding to 0.032 barns/sr/f.u., while the contributions from $\mathrm{O}$ and $\mathrm{H}$ are insignificant [28]. The observed background level in the nuclear scattering is somewhat higher at $0.5-0.8 \mathrm{barns} / \mathrm{sr} / \mathrm{f}$.u., probably due to structural disorder which is commonly observed in goethite [17,23].

We observe an increase in both the spin-incoherent scattering and the nuclear-incoherent scattering at $1.5 \mathrm{~K}$ compared to $300 \mathrm{~K}$. This could be related to inelastic or quasielastic scattering from hydrogen, either in the interior of the particles or on the particle surfaces, moving into the $(Q, E)$ window of the instrument at low temperatures.

The magnetic cross sections are well separated from the other scattering contributions, which was the main objective of the polarized neutron powder diffraction experiment. This enables us to study the magnetic structure of goethite more directly than with unpolarized neutron powder diffraction. The magnetic cross sections show well defined $\{200\}$ and $\{101\}$ diffraction peaks, which will be the basis of our analysis. All samples have significant magnetic cross sections at $300 \mathrm{~K}$ indicating that the Néel temperature is higher than $300 \mathrm{~K}$ even for the 5-nm particles of G1. In all measurements the magnetic spin-flip (SF) and nonspin-flip (NSF) cross sections (not shown) are identical within the error bars. This is expected for a randomly oriented powder of particles with collinear antiferromagnetic order, but not for a powder with preferred orientations. The similarity between SF and NSF cross sections thus shows that no significant macroscopic preferred orientation is present in the powders.

\section{A. Modeling}

The separated magnetic diffraction peaks sit on a flat (almost zero) background and can be modeled well by fitting the data with Voigtian peaks that take the Gaussian instrumental broadening and a Lorentzian sample broadening into account. The fits to the nuclear cross sections obtained at $1.5 \mathrm{~K}$ and the fits to the magnetic cross sections at $1.5 \mathrm{~K}$ and at $150 \mathrm{~K}(\mathrm{G} 1)$ or $300 \mathrm{~K}(\mathrm{G} 2$ and G3) are shown in Fig. 3. The fits were performed in a restricted $q$ range of $0.7 \AA^{-1}-2.1 \AA^{-1}$ for the nuclear cross sections and $0.5 \AA^{-1}-2.0 \AA^{-1}$ for the magnetic cross sections. In this $q$ range the peaks are well enough separated and the background uniform enough to produce good fits.

The fitted part of the nuclear diffraction patterns displays the $\{200\},\{101\}$, and $\{201\}$ peaks as expected. The nuclear scattering looks similar for the three samples with the obvious difference that the peaks of G1 are broader than those of G2 and G3, due to its smaller crystallite size. The nuclear cross sections measured at higher temperatures (not shown) look very similar to the $1.5 \mathrm{~K}$ data, except for a slight decrease in background by less than 0.1 barns/sr/f.u. The fitting function for the nuclear data was a sum of three Voigtian peaks and a sloping background. Fitting the width of both Gaussian and Lorentzian components lead to Gaussian widths that were in some cases smaller than the instrumental resolution. This suggests that there is no significant Gaussian component to the broadening from the sample, and the Gaussian widths of the Voigts were fixed to the instrumental resolution, which was determined from a Gaussian fit of diffraction data from a well-crystallized yttrium iron garnet (YIG) sample, with a linear interpolation of the Gaussian width between the YIG peak positions. The model represents the measured nuclear cross sections well in the fitted $q$ range.

The magnetic cross sections (Fig. 3) are dominated by the strong $\{200\}$ and $\{101\}$ reflections, especially at $1.5 \mathrm{~K}$ where the cross sections could be fitted well with just two Voigtian peaks. At $150 \mathrm{~K}$ for $\mathrm{G} 1$ and $300 \mathrm{~K}$ for $\mathrm{G} 2$ and $\mathrm{G} 3$ the weak $\{001\}$ reflection can be identified as a shoulder between the two more intense peaks. The relative increase of the $\{001\}$ intensity with temperature suggests some degree of spin reorientation. The magnetic cross section of G1 at $300 \mathrm{~K}$ (not shown) has less than half the intensity at $150 \mathrm{~K}$ and the $\{001\}$ peak is no longer detectable. In some of the measured magnetic cross sections there are signs of weak reflections at the $\{201\}$ and $\{010\}$ positions, however, including peaks at these positions did not improve the fits and did not produce meaningful results. The fitted model thus consisted of three Voigtians with Gaussian widths equal to the resolution plus a flat background. In the fit, the position of the $\{001\}$ peak was fixed relative to the $\{200\}$ and $\{101\}$ positions, i.e., $q_{001}=\sqrt{q_{101}^{2}-q_{200}^{2} / 4}$, and its Lorentz width was fixed to the average of the two. The intensities of the fitted peaks were extracted by numerical integration of the Voigt fits and the uncertainties on the intensities were determined by a Monte Carlo approach, i.e., they were determined from a distribution of intensities created from integrating a distribution of Voigts based on the uncertainties on the fit parameters.

\section{B. Structural parameters}

The $a$ and $c$ lattice parameters can be calculated from the positions of the $\{200\}$ and $\{101\}$ peaks. The lattice parameters determined from the nuclear cross sections at $300 \mathrm{~K}$ (Table III ${ }^{3}$ ) are $\approx 0.5 \%$ larger than the values determined with $\mathrm{x}$-ray diffraction [25], probably due to a

\footnotetext{
${ }^{3}$ The uncertainties on experimentally determined values (standard deviations) are given in parenthesis.
}

TABLE III. The $a$ and $c$ lattice parameters determined from fits to the nuclear $\{200\}$ and $\{101\}$ peaks measured at $300 \mathrm{~K}$.

\begin{tabular}{lccc}
\hline \hline & $\mathrm{G} 1$ & $\mathrm{G} 2$ & $\mathrm{G} 3$ \\
\hline$a(\AA)$ & $10.012(7)$ & $9.998(2)$ & $10.001(3)$ \\
$c(\AA)$ & $4.6376(8)$ & $4.6493(3)$ & $4.6409(4)$ \\
\hline \hline
\end{tabular}


small zero point offset of the diffractometer in our experiment. Values determined from the magnetic peaks agree within reason but have a larger uncertainty, in particular at $300 \mathrm{~K}$.

Assuming a Gaussian instrumental resolution [27] and a sample broadening that is Lorentzian and caused by finite size effects only, the magnetic and nuclear correlation lengths in the direction along $\mathbf{q}_{\mathbf{h k l}}$ can be estimated from the fitted Lorentz widths through

$$
d_{h k l}=2 \pi K / \Delta q_{\text {Lorz, } h k l},
$$

where $\Delta q_{\text {Lorz, hkl }}$ is the Lorentzian FWHM of the Voigtian peak and $K$ is the Scherrer constant, which typically takes values between 0.7 and 1 , depending on the shape of the crystallites/domains. Because any strain contribution to the peak broadening is neglected, the sizes determined through Eq. (9) will be a lower limit. However, the strain contribution is not likely to be very significant, as the broadening from the finite particle size is substantial. The nuclear and magnetic correlation lengths in the [100] and [101] directions determined from the fits of the $\{200\}$ and $\{101\}$ peaks assuming $K=1$ (approximately true for a spherical shape) are shown in Fig. 4.

Within the error bars the nuclear correlation lengths are constant with temperature and taking a temperature average we get [100] correlation lengths of 4.6 (3) nm, 13.6 (3) nm, and 13.7 (8) $\mathrm{nm}$ for samples G1, G2, and G3, respectively. Likewise, the temperature averaged nuclear correlation lengths in the [101] directions are 6.14 (2) $\mathrm{nm}, 9.68$ (3) $\mathrm{nm}$, and 12.5 (6) $\mathrm{nm}$. The magnetic correlation lengths determined from the broadening of the magnetic $\{200\}$ peak, on the other hand, decrease with increasing temperature. For G2 and G3 the magnetic [100] correlation lengths at $1.5 \mathrm{~K}$ are approximately $3 \mathrm{~nm}$ shorter than the nuclear correlation lengths. For G1 the [100] magnetic correlation length at $1.5 \mathrm{~K}$ and $150 \mathrm{~K}$ is actually slightly $(\approx 1 \mathrm{~nm}$ ) longer than the nuclear. In the [101] direction the magnetic and nuclear correlation lengths are very similar. If it is assumed that the particle shape has the same symmetry as the unit cell, the correlation length in the [001] direction can be determined as $d_{N, 001}=d_{N, 101} \cos \gamma$, resulting in $5.57(2) \mathrm{nm}, 8.78$ (3) nm, and 11.3 (6) $\mathrm{nm}$ for the three samples.

\section{Magnetic structure}

The magnetic moment perpendicular to $q_{200}$ and $q_{101}$ was calculated from the ratios of the measured magnetic and nuclear intensities using Eq. (5). $M_{\perp, 200}$ and $M_{\perp, 101}$ are displayed in Fig. 5 (left). If the spins were confined to be along the $b$ axis, $M_{\perp, 200}$ and $M_{\perp, 101}$ would be equal. Instead, we see that $M_{\perp, 200}$ is significantly larger than $M_{\perp, 101}$ for all three samples and at both low and high temperatures, which means that the spins cannot be confined to be along [010]. At $1.5 \mathrm{~K}, M_{\perp, 200}$ is the same for all three samples within the uncertainty (2.21 (3) $\mu_{\mathrm{B}}$ for G1, 2.24 (2) $\mu_{\mathrm{B}}$ for $\mathrm{G} 2$, and 2.25 (2) $\mu_{\mathrm{B}}$ for G3) and $M_{\perp, 101}$ is also very similar between the samples (1.89 (2) $\mu_{\mathrm{B}}$ for $\mathrm{G} 1,1.942$ (15) $\mu_{\mathrm{B}}$ for $\mathrm{G} 2$, and $1.96(2) \mu_{\mathrm{B}}$ for G3). The magnetic moments decrease with increasing temperature in a similar fashion for all three samples with $M_{\perp, 200} \approx 1.4 \mu_{\mathrm{B}}$ and $M_{\perp, 101} \approx 1.1 \mu_{\mathrm{B}}$ at $300 \mathrm{~K}$. Shown in Fig. 5 (right) is the ratio between $M_{\perp, 200}$ and $M_{\perp, 101}\left(M_{\perp, \text { rel }}\right)$ as determined from Eq. (5) as well as directly from the ratio between the measured magnetic cross sections through Eq. (6).
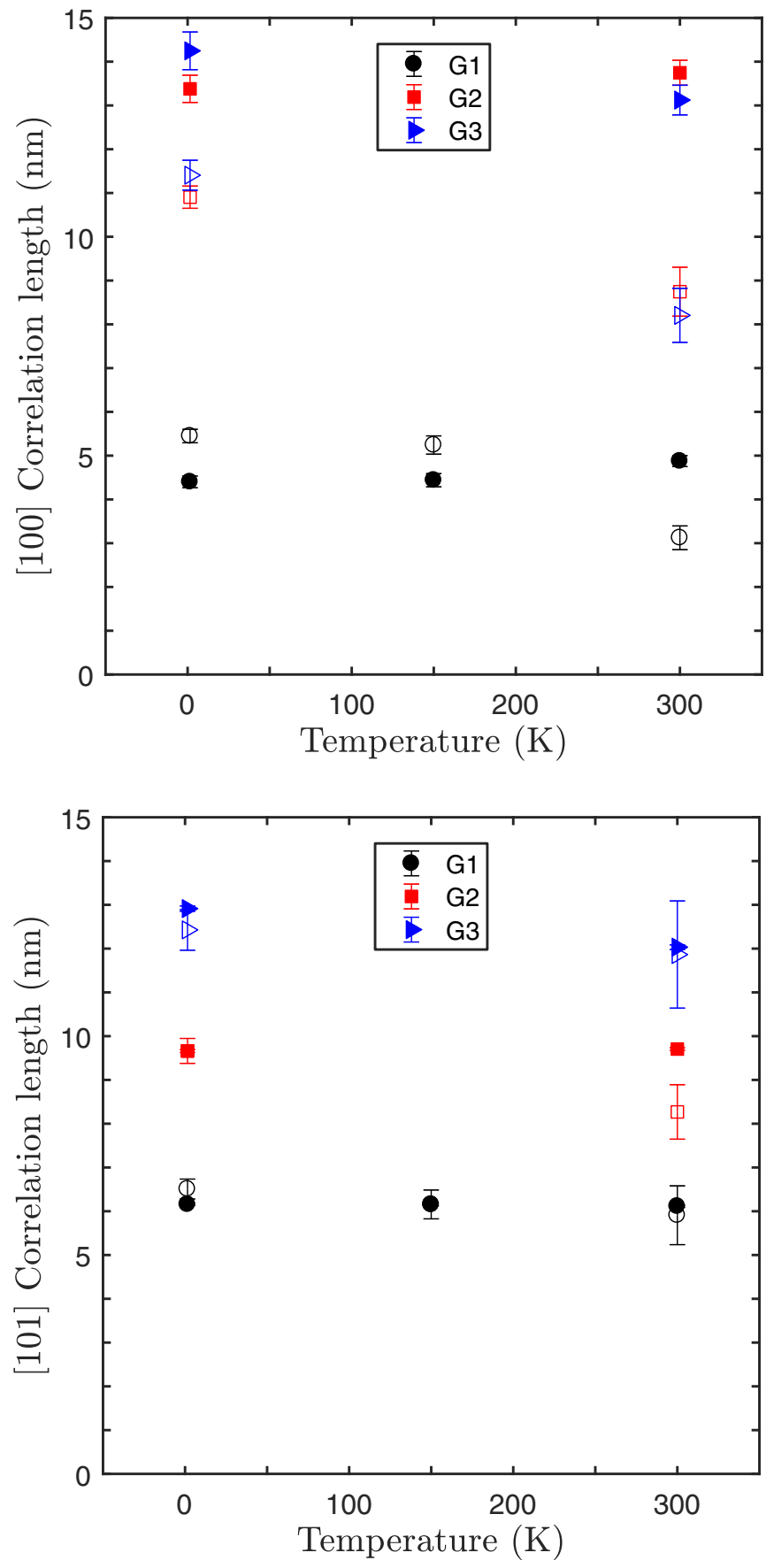

FIG. 4. Correlation lengths in the [100] (top) and [101] (bottom) directions determined from Lorentzian widths of fits to the diffraction peaks. Open symbols are magnetic correlation lengths and filled symbols are nuclear correlation lengths. The error bars representing one standard deviation are in some cases smaller than the size of the points.

At $1.5 \mathrm{~K}, M_{\perp, \text { rel }} \approx 1.15$ for all samples regardless of which of the two normalization methods is used. For G1, the ratio is increased at $300 \mathrm{~K}$, however, the uncertainty on this point is large because the magnetic cross section is close to the background and not fitted well. The changes in the ratio for $\mathrm{G} 2$ and $\mathrm{G} 3$ are small.

The spin angles with respect to [010] are calculated from Eq. (8) using $M_{\perp, \text { rel }}$ determined from the magnetic intensities 

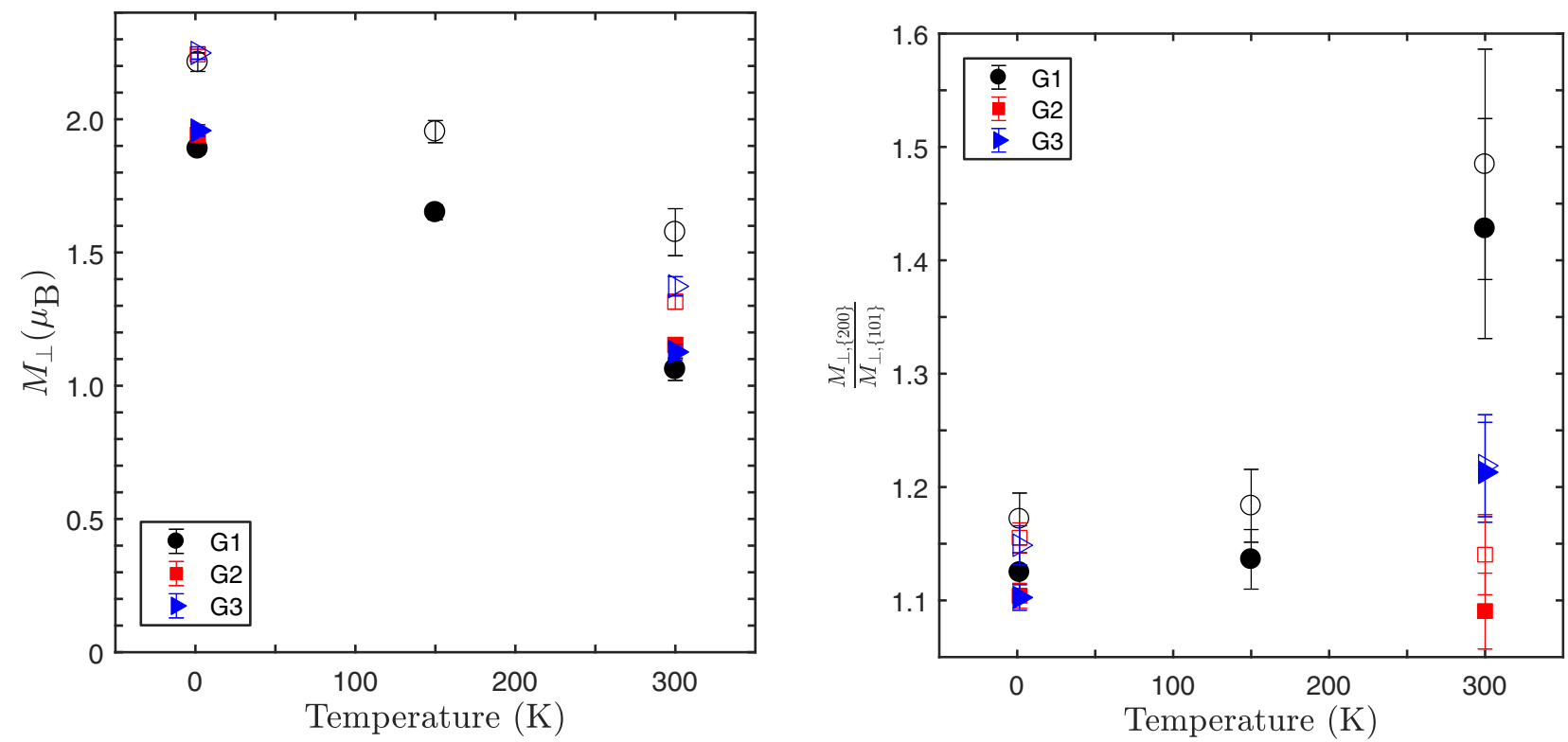

FIG. 5. Left: Magnetic moment calculated from measured intensities using Eq. 5. Open symbols show the magnetic moment perpendicular to [100] and the filled symbols show the magnetic moment perpendicular to [101]. Right: $M_{\perp, \text { rel }}=M_{\perp, 200} / M_{\perp, 101}$ determined from the measured $M_{\perp, 200}$ and $M_{\perp, 101}$ obtained by normalizing the magnetic cross sections to the nuclear cross sections (open symbols) and directly from the ratio of the magnetic cross sections (filled symbols). The error bars represent one standard deviation and are smaller than the size of the points in some cases.

through Eq. (6), and are are shown in Fig. 6. At $1.5 \mathrm{~K}$, the out-of-plane spin angles are $\alpha=30(2)^{\circ}, \alpha=27.9(13)^{\circ}$, and $\alpha=27.7(14)^{\circ}$ for samples G1, G2, and G3, respectively. For $\mathrm{G} 1, \alpha$ is $32(3)^{\circ}$ at $150 \mathrm{~K}$ and increases to $52(5)^{\circ}$ at $300 \mathrm{~K}$. For G2 and G3, the spin angles at $300 \mathrm{~K}$ are $\alpha=26(4)^{\circ}$ and $\alpha=39(3)^{\circ}$, respectively.

\section{DISCUSSION}

Polarized neutron powder diffraction allows us to separate the magnetic cross sections from the nuclear and spin-incoherent cross sections. This enables an analysis of the magnetic structure of goethite nanoparticles that would be difficult with unpolarized neutron powder diffraction because of the overlap of nuclear and magnetic diffraction peaks. The magnetic cross sections show us that the antiferromagnetic order persists at temperatures up to $300 \mathrm{~K}$ even in the $\mathrm{G} 1$ sample, which has a particle size of only about $5 \mathrm{~nm}$.

In Mössbauer spectroscopy measurements, the magnetic splitting of the spectra of G1 was previously found to be collapsed at $260 \mathrm{~K} \mathrm{[22].} \mathrm{The} \mathrm{fact} \mathrm{that} \mathrm{the} \mathrm{magnetic} \mathrm{cross}$ sections persist at $300 \mathrm{~K}$ confirms the hypothesis that the collapse of the Mössbauer lines is due to superparamagnetic relaxation on a time scale faster than Mössbauer spectroscopy $\left(\approx 10^{-9} \mathrm{~s}\right)$ but slower than neutron diffraction $\left(\approx 10^{-12} \mathrm{~s}\right)$ and not due to a reduced Néel temperature.

The G1 sample is known from TEM and x-ray diffraction studies to consist of roughly spherical particles with a particle size of about $5 \mathrm{~nm}$ and approximately the same crystalline size [22,25]. The correlation lengths we determine from the broadening of the nuclear diffraction peaks are in good agreement with these studies. G2 and G3 are known to consist of significantly larger elongated particles that consist of several grains. The x-ray results [25] show that the crystallites (grains) are elongated in the [010] direction but are of roughly equal length in the [100] and [001] directions. We do not have access to a well-separated reflection with a scattering vector component in the [010] direction and can thus only determine correlation lengths in the $a c$ plane. We find that both G2 and G3 are slightly longer along [100] than along [001]. The nuclear correlation lengths we find for G2 and G3 are significantly larger than the reported $\mathrm{x}$-ray results. A reason for this discrepancy could be a difference in the value used for the Scherrer constant (in principle different values of $K$ should be used for elongated and spherical particles), or an underestimated instrumental broadening in the reference [25] or an overestimate in our analysis. Such a systematic error would not affect the relationship between different determined correlation lengths but only their absolute values. Any effect on the determined intensities derived from an error on the instrumental resolution would be small and is thus not expected to influence the determination of the spin orientation. For G2 and $\mathrm{G} 3$, the magnetic correlation lengths at $1.5 \mathrm{~K}$ are similar to the nuclear correlation lengths in the [001] direction, but in the [100] direction, which is the direction of antiferromagnetic modulation, the magnetic correlation lengths are approximately $3 \mathrm{~nm}$ smaller than the nuclear correlation lengths. This can be explained by breakdown of the antiferromagnetic order near the [100] faces of the crystallites but also by some particles having multiple antiferromagnetic domains. A magnetically disordered surface layer has been suggested by many authors to explain the magnetic properties of magnetic nanoparticles, such as the decreased magnetization of ferromagnetic materials $[29,30]$ and the net magnetization of antiferromagnetic materials [22,31-33] when the particle size is decreased to the nanoscale. However, few direct measurements of a disordered 
particle shell have been performed [34,35]. For G1, the magnetic correlation length in the [100] direction is about $1 \mathrm{~nm}$ longer than the nuclear at $1.5 \mathrm{~K}$ and $150 \mathrm{~K}$, which can probably be ascribed to a slight misfit of the nuclear $\{200\}$ peak. In the [001] direction, the magnetic and nuclear correlation lengths are identical. The apparent decrease of the magnetic [100] correlation lengths with increasing temperature could indicate that the antiferromagnetic order further shrinks, however, it is perhaps more likely to attribute the increase in peak broadening to inelastic magnetic scattering.

The measured magnetic cross sections normalized to the nuclear intensities were used to determine the size of the magnetic moment perpendicular to the [100] and [101] scattering vectors. At $1.5 \mathrm{~K}$ the size of the magnetic moments is on average 2.24 (2) $\mu_{\mathrm{B}}$ perpendicular to [100] and 1.93 (4) $\mu_{\mathrm{B}}$ perpendicular to [101], with insignificant variation between samples. Provided the spin orientation is confined to the $b c$ plane, the total moment in our samples is equal to the 2.24 (2) $\mu_{\mathrm{B}}$ perpendicular to [100]. If the spins have a component along the $a$ axis, the total moment will be higher. Earlier studies have consistently found the $\mathrm{Fe}^{3+}$ magnetic moment in goethite to be lower than the $5 \mu_{\mathrm{B}}$ expected for high spin $\mathrm{Fe}^{3+}$, in particular in particles with a small crystallite size. For example, Zepeda-Alarcon et al. [18] finds a $\mathrm{Fe}^{3+}$ magnetic moment of 4.45 (3) $\mu_{\mathrm{B}}$ for a sample with a crystallite size of 96 (3) $\mathrm{nm}$ and 3.04 (13) $\mu_{\mathrm{B}}$ for a sample a crystallite size of 12.2 (6) $\mathrm{nm}$. The reduced magnetic moment in goethite can be attributed to magnetic disorder at the grain interfaces as well as structural defects in the interior of the grains.

The difference between $M_{\perp, 200}$ and $M_{\perp, 101}$, which is consistently about $15 \%$ for all samples at $1.5 \mathrm{~K}$, clearly indicates that the magnetic moment is not parallel to the $b$ axis and has some component parallel to the $c$ axis. The $P 2_{1}^{\prime} / c$ space group allows a tilt towards the $a$ axis as well as towards the $c$ axis, and our experiment cannot rule out a spin component along the $a$ axis. However, the very low intensity of the $\{201\}$ reflection shows that the main tilt is towards the $c$ axis. If the spin rotation is entirely in the $b c$ plane, as suggested by Coey et al. [19], the off-axis spin angle can be calculated from Eq. (8), as shown in Fig. 6. The off-axis spin angles of $\alpha=27.7(14)^{\circ}-30(2)^{\circ}$ at $1.5 \mathrm{~K}$ is significantly larger than the $13^{\circ}$ canting angle found by Coey et al. [19]. For G1, which is the sample with $\approx 5 \mathrm{~nm}$ particle size, the spins are further reoriented to an angle of $52(5)^{\circ}$ at $300 \mathrm{~K}$. For G2, there is no significant change in $\alpha$ with temperature, whereas G3 shows a small increase to $\alpha=39(3)^{\circ}$ at $300 \mathrm{~K}$. Further evidence for a spin reorientation is found in the increasing relative intensity of the magnetic $\{001\}$ peak with increasing temperature. However, this spin reorientation would have to be in the $a c$ plane in addition to the rotation away from the $b$ axis.

With a spin component perpendicular to [010], the magnetic $\{010\}$ peak should have a nonzero intensity at $q=2.081 \AA^{-1}$, and evidence of a weak reflection can be seen at this position (especially for G1), although it is certainly too weak for any quantitative analysis. If the spins are in the $b c$ plane, the $\{010\}$ peak is expected to have an intensity that is approximately $13 \%$ that of the $\{101\}$ peak, which would indicate that it should be detectable, albeit not much larger than the fluctuations in the background. The fact that the $\{010\}$ peak appears slightly less intense than expected on the basis of the spin-angle determined

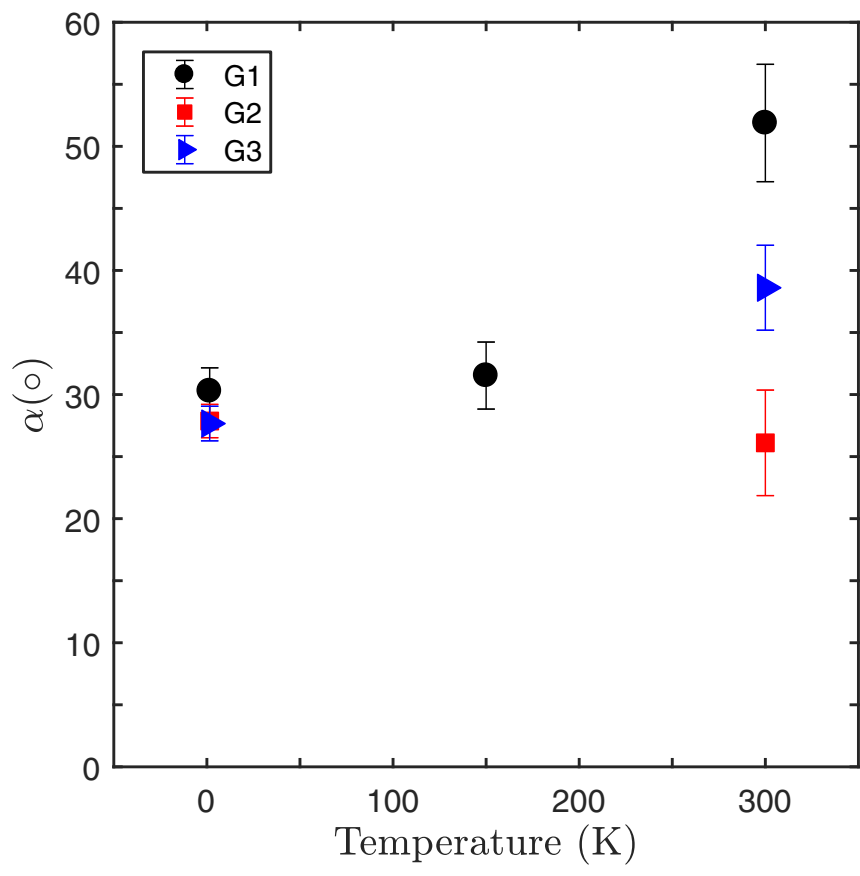

FIG. 6. Spin angle with respect to [010] calculated from Eq. (8). The error bars represent one standard deviation.

from the ratio of the two main peaks could suggest that the spin angles are somewhat overestimated due to systematic errors. This means that the values determined for $\alpha$ should perhaps not be considered as precise values, but the nonzero value of $\alpha$ remains significant, and is confirmed by the faint but visible $\{010\}$ reflection.

The spin orientation in goethite could depend on the size of the crystallographic grains as well as the presence of impurities or defects in the structure, and could thus be sample dependent. Goethite usually occurs in nanocrystalline form and often has defects, such as deviations from stoichiometry, and our study of goethite nanoparticles of varying grain size show that the spins cannot generally be assumed to be confined to the crystallographic $b$ axis.

In magnetic nanoparticles, the spins can both be conceived to have an overall canting due to surface contributions to the magnetic anisotropy and localized canting of spins near the particle surface or defects in the interior of the particles [36]. Our studies determine the average spin orientation and does not, as such, distinguish between these two scenarios.

The observed spin reorientation at $300 \mathrm{~K}$ could be due to different temperature dependence of different spin populations, e.g., frustrated surface spins and spins in well-defined antiferromagnetic surroundings [37].

\section{CONCLUSIONS}

By using polarized neutron powder diffraction on nanocrystalline goethite powders, we find a significant off-axis spin component in contrary to the usual assumed structure with the antiferromagnetic spins confined to the $b$ axis. Assuming the spins to be confined to the $b c$ plane, the spin rotation away from the $b$ axis is $30(2)^{\circ}$ at $1.5 \mathrm{~K}$, for a sample 
of 5-nm goethite particles, and $27.9(13)^{\circ}$ and $27.7(14)^{\circ}$ for two samples of larger goethite nanoparticles. For the 5-nm particles, the spins rotate to even larger off-axis angles at $300 \mathrm{~K}$. Our results, as well as the results of Coey et al. [19], clearly show that goethite can have a significant off-axis spin component and that the conventionally assumed magnetic structure cannot automatically be assumed to be correct. For the two samples with the larger multigrain particles, we find a magnetic correlation length in the [100] direction of antiferromagnetic modulation that is $3 \mathrm{~nm}$ smaller than the nuclear correlation length. This is most likely a direct observation of a magnetically disordered layer at the (100) faces of the crystallites.

\section{ACKNOWLEDGMENTS}

The authors gratefully acknowledge the access to neutron scattering experiments at the D7 instrument at ILL, Grenoble. This work was in part supported by the Danish Council of independent Research FNU, through DANSCATT.
[1] J. Nogués and I. K. Schuller, J. Magn. Magn. Mater. 192, 203 (1999).

[2] V. Skumryev, S. Stoyanov, Y. Zhang, G. Hadjipanayis, D. Givord, and J. Nogués, Nature 423, 850 (2003).

[3] V. Schneider, A. Reinholdt, U. Kreibig, T. Weirich, G. Güntherodt, B. Beschoten, A. Tillmanns, H. Krenn, K. Rumpf, and P. Granitzer, Z. Phys. Chem. 220, 173 (2006).

[4] I. Groza, R. Morel, A. Brenac, C. Beigné, and L. Notin, IEEE Trans. Magn. 47, 3355 (2011).

[5] M. Estrader, A. López-Ortega, S. Estradé, I. V. Golosovsky, G. Salazar-Alvarez, M. Vasilakaki, K. N. Trohidou, M. Varela, D. C. Stanley, M. Sinko, M. J. Pechan, D. J. Keavney, F. Peiró, S. Suriñach, M. D. Baró, and J. Nogués, Nat. Commun. 4, 2960 (2013).

[6] K. L. Krycka, J. A. Borchers, M. Laver, G. Salazar-Alvarez, A. López-Ortega, M. Estrader, S. Suriñach, M. D. Baró, J. Sort, and J. Nogués, J. Appl. Phys. 113, 17B531 (2013).

[7] K. Chakrabarti, B. Sarkar, V. Dev Ashok, K. Das, S. Sinha Chaudhuri, A. Mitra, and S. K. De, J. Appl. Phys. 115, 013906 (2014).

[8] A. Roy, J. A. De Toro, V. S. Amaral, P. Muniz, J. M. Riveiro, and J. M. F. Ferreira, J. Appl. Phys. 115, 073904 (2014).

[9] J. A. De Toro, D. P. Marques, P. Muñiz, V. Skumryev, J. Sort, D. Givord, and J. Nogués, Phys. Rev. Lett. 115, 057201 (2015).

[10] M. Vasilakaki, K. N. Trohidou, and J. Nogués, Sci. Rep. 5, 9609 (2015).

[11] R. H. Kodama, A. E. Berkowitz, E. J. McNiff, and S. Foner, Phys. Rev. Lett. 77, 394 (1996).

[12] L. Berger, Y. Labaye, M. Tamine, and J. M. D. Coey, Phys. Rev. B 77, 104431 (2008).

[13] K. L. Krycka, R. A. Booth, C. R. Hogg, Y. Ijiri, J. A. Borchers, W. C. Chen, S. M. Watson, M. Laver, T. R. Gentile, L. R. Dedon, S. Harris, J. J. Rhyne, and S. A. Majetich, Phys. Rev. Lett. 104, 207203 (2010).

[14] E. Brok, K. Lefmann, P. P. Deen, B. Lebech, H. Jacobsen, G. J. Nilsen, L. Keller, and C. Frandsen, Phys. Rev. B 91, 014431 (2015).

[15] A. Szytula, A. Burewicz, Z. Dimitijevic, S. Krasnicki, H. Rzany, J. Todorovic, A. Wanic, and W. Wolski, Phys. Status Solidi 26, 429 (1968)

[16] S. Bocquet and S. J. Kennedy, J. Magn. Magn. Mater. 109, 260 (1992).

[17] C. A. Barrero, J. D. Betancur, J. M. Greneche, G. F. Goya, and T. S. Berquó, Geophys. J. Int. 164, 331 (2006).

[18] E. Zepeda-Alarcon, H. Nakotte, A. F. Gualtieri, G. King, K. Page, S. C. Vogel, H.-W. Wang, and H.-R. Wenk, J. Appl. Crystallogr. 47, 1983 (2014).
[19] J. Coey, A. Barry, J.-M. Brotto, H. Rakato, S. Brennan, W. N. Mussel, A. Collomb, and D. Fruchart, J. Phys.: Condens. Matter 7, 759 (1995).

[20] J. B. Forsyth, J. G. Hedley, and C. E. Johnson, J. Phys. C 1, 179 (1968).

[21] D. E. Madsen, L. Cervera-Gontard, T. Kasama, R. E. DuninBorkowski, C. B. Koch, M. F. Hansen, C. Frandsen, and S. Mørup, J. Phys.: Condens. Matter 21, 016007 (2009).

[22] E. Brok, C. Frandsen, D. E. Madsen, H. Jacobsen, J. O. Birk, K. Lefmann, J. Bendix, K. S. Pedersen, C. B. Boothroyd, A. A. Berhe, G. G. Simeoni, and S. Mørup, J. Phys. D 47, 365003 (2014).

[23] Q. A. Pankhurst, L. F. Barquín, J. S. Lord, A. Amato, and U. Zimmermann, Phys. Rev. B 85, 174437 (2012).

[24] G. L. Squires, Thermal Neutron Scattering (Cambridge University Press, Cambridge, 1978).

[25] C. Frandsen, D. E. Madsen, C. B. Boothroyd, and S. Mørup, Croat. Chem. Acta 88, 481 (2015).

[26] J. R. Stewart, P. P. Deen, K. H. Andersen, H. Schober, J.-F. Barthélémy, J. M. Hillier, A. P. Murani, T. Hayes, and B. Lindenau, J. Appl. Crystallogr. 42, 69 (2008).

[27] T. Fennell, L. Mangin-Thro, H. Mutka, G. Nilsen, and A. Wildes, Nucl. Instrum. Methods Phys. Res., Sect. A: Accelerators, Spectrometers, Detectors and Associated Equipment 857, 24 (2017).

[28] Edited by A.-J. Dianoux and G. Lander, Neutron Data Booklet (Institut Laue-Langevin, Grenoble, 2003).

[29] J. Curiale, M. Granada, H. E. Troiani, R. D. Sánchez, A. G. Leyva, P. Levy, and K. Samwer, Appl. Phys. Lett. 95, 043106 (2009).

[30] P. Dutta, S. Pal, M. S. Seehra, N. Shah, and G. P. Huffman, J. Appl. Phys. 105, 07B501 (2009).

[31] S. Mandal, S. Banerjee, and K. S. R. Menon, Phys. Rev. B 80, 214420 (2009).

[32] M. Tadic, M. Panjan, D. Markovic, B. Stanojevic, D. Jovanovic, I. Milosevic, and V. Spasojevic, J. Alloys Compd. 586, S322 (2014).

[33] R. N. Bhowmik, R. Nagarajan, and R. Ranganathan, Phys. Rev. B 69, 054430 (2004).

[34] D. Lin, A. C. Nunes, C. F. Majkrzak, and A. E. Berkowitz, J. Magn. Magn. Mater. 145, 343 (1995).

[35] J. F. K. Cooper, A. Ionescu, R. M. Langford, K. R. A. Ziebeck, C. H. W. Barnes, R. Gruar, C. Tighe, J. A. Darr, N. T. K. Thanh, and B. Ouladdiaf, J. Appl. Phys. 114, 083906 (2013).

[36] R. H. Kodama, J. Magn. Magn. Mater. 200, 359 (1999).

[37] H. Jacobsen, K. Lefmann, E. Brok, C. Frandsen, and S. Mørup, J. Magn. Magn. Mater. 324, 3218 (2012). 